\title{
Diagnosis of ovarian cancers using thoracoscopy: Three case reports and review of the literature
}

\author{
MAKOTO NAKAO, TETSUYA OGURI, MIKINORI MIYAZAKI, TAKEHIRO UEMURA, \\ OSAMU TAKAKUWA, EIJI KUNII, HIROTSUGU OHKUBO, KEN MAENO and SHIGEKI SATO \\ Department of Medical Oncology and Immunology, Nagoya City University, \\ School of Medical Sciences, Nagoya 467-8601, Japan
}

Received February 24, 2012; Accepted April 2, 2012

DOI: $10.3892 /$ etm.2012.541

\begin{abstract}
Thoracoscopy is a useful method for diagnosing plural lesions. We diagnosed 3 cases of ovarian adenocarcinoma by thoracoscopy. All patients were middle-aged and elderly women. Initial examination revealed intrathoracic lesions including pleural effusion, but intraperitoneal lesions were unclear. In all cases, adequate samples of tumor cells obtained using thoracoscopy were used in the identification of tumor origin. Elevated serum levels of cancer antigen 125 (CA-125) and Krebs von den lungen-6 (KL-6) were observed in 2 of the 3 patients. Diagnoses of ovarian cancer were based on immunohistochemical examinations and clinical course. This report describes the diagnostic usefulness of thoracoscopy and serum KL-6 in cases of ovarian cancer with predominantly intrathoracic lesions.
\end{abstract}

\section{Introduction}

Thoracoscopy is a useful technique for diagnosing plural lesions, and it provides valuable information for identifying a cancer of an unknown primary origin (CUP) (1-3). The diagnostic procedure for the evaluation of CUP includes clinical and laboratory investigations, namely, imaging, endoscopy, pathology and tumor markers (4). In particular, in the work-up of a CUP patient with exudative pleural effusion, thoracoscopy is an established tool to aid diagnosis $(5,6)$.

Ovarian cancer with intrathoracic metastases is often accompanied by abdominal-related symptoms, and ovarian cancer cases with only thoracic lesions are rare (7-10). It is not uncommon to initially categorize these rare cases as CUP $(4,9,10)$. Here, we report 3 cases of ovarian cancer with

Correspondence to: Dr Tetsuya Oguri, Department of Medical Oncology and Immunology, Nagoya City University, School of Medical Sciences, 1 Kawasumi, Mizuho-cho Mizuho-ku, Nagoya 467-8601, Japan

E-mail: t-oguri@med.nagoya-cu.ac.jp

Key words: thoracoscopy, ovarian cancer, Krebs von den lungen-6 predominantly intrathoracic lesions, which were diagnosed by thoracoscopy. In addition, in these cases, we observed an association between the serum Krebs von den lungen-6 (KL-6) level and the state of the ovarian cancer.

\section{Case reports}

Case 1. A 46-year-old woman was referred to our hospital with right pleural effusion on chest radiograph and computed tomography (CT) (Fig. 1A and B). Her chief complaints were coughing and exertional dyspnea. She underwent diagnostic thoracentesis and cytological examination of pleural effusion showed adenocarcinoma cells. We could not detect the origin of the adenocarcinoma on chest and abdominal CT, gastroscopy, physical examination and gynecological examination. Serological test revealed highly elevated levels of cancer antigen 125 (CA-125) (761.6 U/ml; normal <28) and KL-6 (6991 U/ml; normal <500). Thoracoscopy was performed under local anesthesia, and multiple small nodular lesions of parietal pleura were observed (Fig. 1C). Histopathological and immunohistochemical analyses of pleural lesions showed poorly differentiated adenocarcinoma originating in the ovary or endometrium; the lesion was positive for CA-125, cytokeratin (CK) 7, epithelial membrane antigen (EMA), and vimentin, and negative for thyroid transcription factor (TTF)-1, CK20, calretinin, carcinoembryonic antigen (CEA) and gross cystic disease fluid protein (GCDFP)-15 (Fig. 1D and E). Magnetic resonance imaging (MRI) suggested the possibility of an ovarian tumor. She received chemotherapy involving paclitaxel plus carboplatin and abdominal surgery. She achieved a good response, and the serum levels of CA-125 and KL-6 decreased to normal levels (7.9 and $284 \mathrm{U} / \mathrm{ml}$, respectively). Her final diagnosis was stage IV ovarian serous papillary carcinoma.

Case 2. A 50-year-old woman was admitted to our hospital with complaints of coughing and dyspnea. Chest radiograph and $\mathrm{CT}$ indicated right pleural effusion (Fig. 2A and B), and a cytological examination of the pleural effusion suspected cancerous cells. We were unable to determine the origin of the cancer on chest and abdominal $\mathrm{CT}$, positron emission tomography (PET)-CT, physical examination, and gynecological examination. Serological test revealed elevated levels 

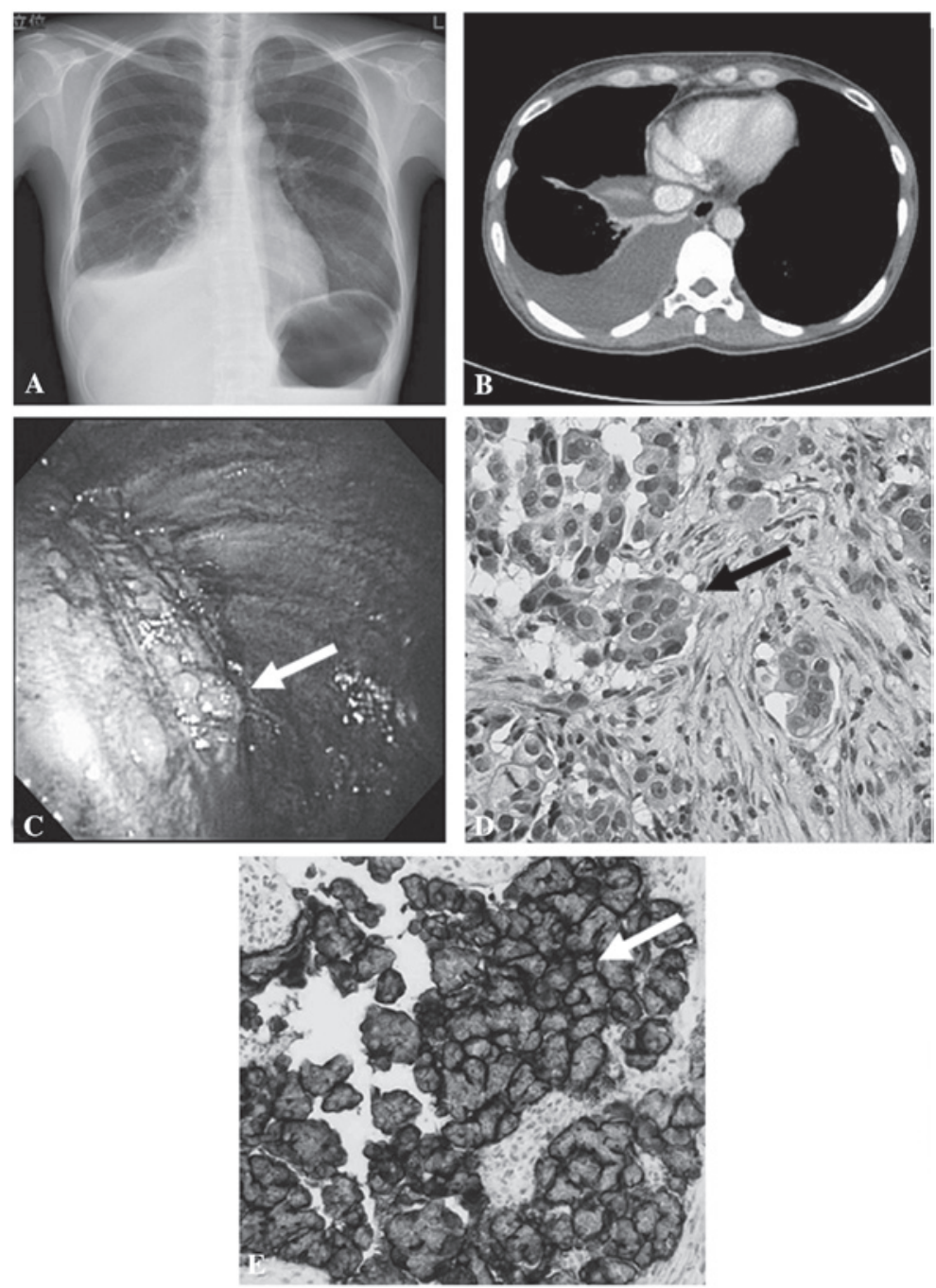

Figure 1. Case 1. (A) Chest X-ray film showing right pleural effusion. (B) CT scan showing right pleural effusion. (C) Thoracoscopic image showing multiple small nodular lesions of parietal pleura (diaphragmatic part). (D) A biopsy specimen from a pleural lesion. Hematoxylin and eosin staining (x400). (E) A biopsy specimen from a pleural lesion. Immunohistochemical staining with anti-CA-125 is positive (x400).

of CA-125 (211.1 U/ml) and KL-6 (791 U/ml). Thoracoscopy under general anesthesia showed multiple small nodular lesions of the parietal pleura and histopathological analyses of pleural lesions showed adenocarcinoma (Fig. 2C). Since we suspected lung adenocarcinoma or CUP, the patient received 4 courses of gemcitabine plus cisplatin. She achieved a good response, and the serum levels of CA-125 and KL-6 were decreased (13 and $490 \mathrm{U} / \mathrm{ml}$, respectively). Approximately 2 years following the initial examination, she was admitted to our hospital with a complaint of abdominal fullness. Although chest $\mathrm{CT}$ showed no thoracic lesion, abdominal CT indicated ascites and a pelvic mass involving the left ovary. Serological test revealed re-elevated level of CA-125 (122.1 U/ml). To identify the origin of the previously diagnosed cancer, we re-examined the specimens of pleural lesions. Immunohistochemical staining revealed that the samples were positive for CA-125 and CK7, and negative for TTF-1, CK20 and calretinin (Fig. 2D and E). Taking into account both immunohistochemical analyses and her clinical course, the patient was diagnosed with ovarian adenocarcinoma.
Case 3. An 81-year-old woman with a complaint of dyspnea was referred to our hospital with pleural effusion on the chest radiograph and CT (Fig. 3A and B). Cytological examination of pleural effusion showed carcinoma cells. Serological examination revealed slightly elevated CA-125 $(34.5 \mathrm{U} / \mathrm{ml})$ and normal KL-6 $(129 \mathrm{U} / \mathrm{ml})$. We were unable to detect the origin of the carcinoma cells on chest and upper abdominal CT, physical examination and gynecological examination at the initial visit. Thoracoscopy under local anesthesia showed no lesion of the parietal pleura, and biopsies of the pleura showed normal tissue (Fig. 3C). Further analysis of the pleural fluid obtained by thoracoscopy suggested adenocarcinoma originating in the ovary; immunohistochemical examination of a pleural fluid cell block showed positive results for CA-125, CK7, CA19-9, CEA, epithelial antigen (Ber-EP4), and vimentin, and negative results for TTF-1, CK20, calretinin, CK5/6, caudal-type homeobox transcription factor-2 (CDX-2) and GCDFP-15 (Fig. 3D and E). PET-CT revealed a pelvic tumor involving the left ovary and small peritoneal dissemination. Therefore, the patient was diagnosed with stage IV ovarian adenocarcinoma. 

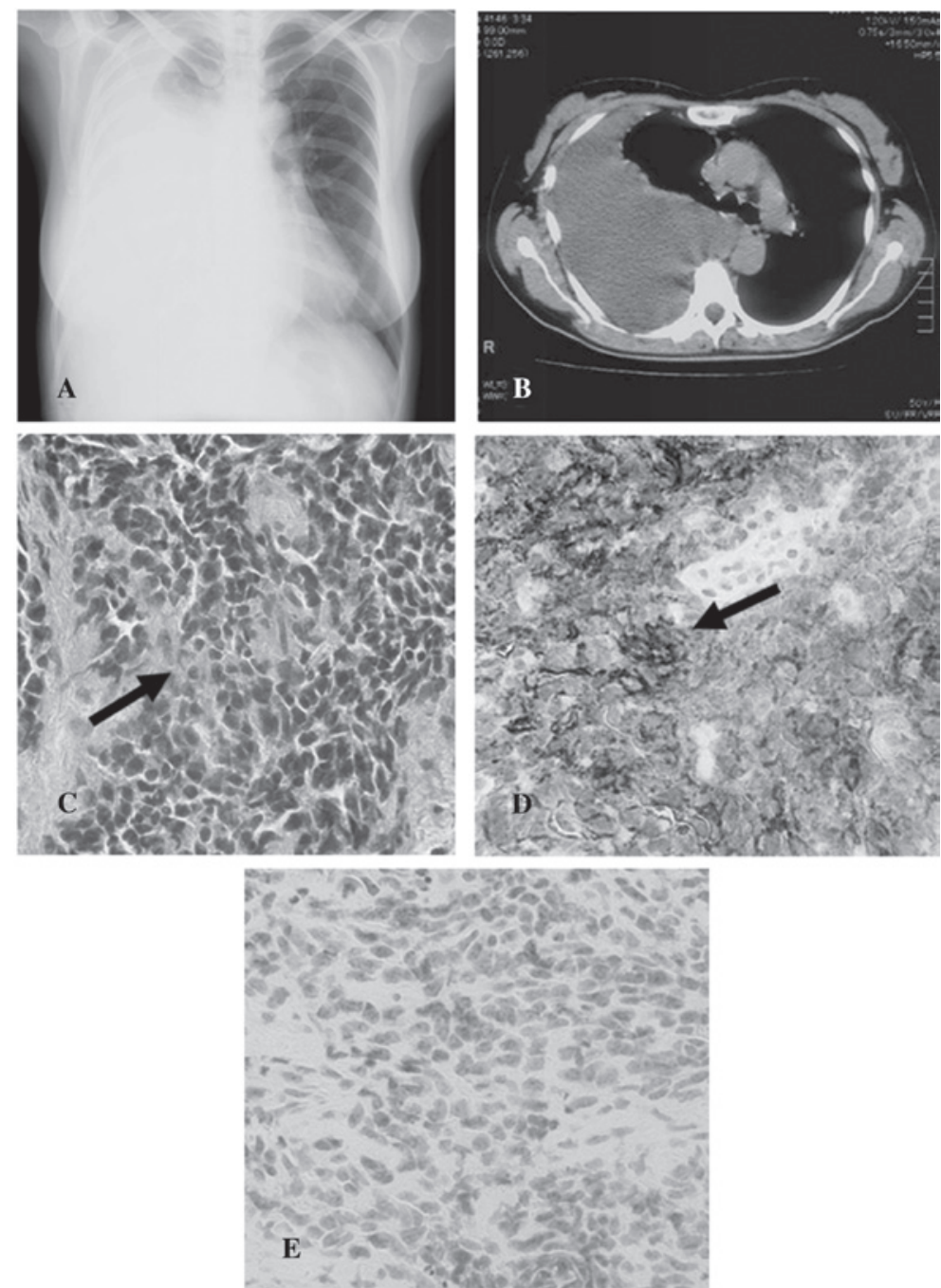

Figure 2. Case 2. (A) Chest X-ray film showing right pleural effusion. (B) CT scan showing right pleural effusion. (C) A biopsy specimen from a pleural lesion Hematoxylin and eosin staining (x400). (D) Immunohistochemical staining of a biopsy specimen showed positivity for CA-125 (x400). (E) Immunohistochemical staining of a biopsy specimen indicating negativity for TTF-1 (x400).

\section{Discussion}

We report three cases of ovarian adenocarcinomas diagnosed by thoracoscopy. All of the patients were middle-aged and elderly women with pleural effusion. Two of the three patients underwent thoracoscopy under local anesthesia and one patient underwent surgery under general anesthesia. Elevated levels of serum CA-125 and KL-6 were observed in two patients, and these markers indicated the state of the disease.

Diagnostic thoracoscopy under local anesthesia is a safe and effective technique in the work-up of a patient with pleural effusion of CUP (1-3). In Western countries, thoracoscopy under local anesthesia is called medical thoracoscopy, and it is an established tool used to aid diagnosis, particularly in patients with exudative pleural effusion of unclear origin $(5,6)$. Diagnostic medical thoracoscopy for pleural effusions can be used to identify pleural lesions caused by malignancy with $>90 \%$ precision, and pathological examination of biopsy specimens provides useful information for the diagnosis of primary lesions (2). Herein, we describe two patients with exudative pleural effusion who were accurately and safely diagnosed with ovarian cancer by medical thoracoscopy. Since medical thoracoscopy is relatively easily performed by pulmonologists, further utilization of this technique should be promoted at Japanese clinical sites.

The most common extra-abdominal site of ovarian cancer is the pleural space, and lung parenchymal involvement is relatively rare (11). In numerous ovarian cancer cases, abdominal symptoms are usually predominant and cases with only intrathoracic lesions are rare $(9,11)$. Ovarian cancer cases with isolated malignant pleural effusion are likely to be categorized as CUP at the time of initial diagnosis $(4,9)$. In previous reports, in approximately $10-15 \%$ of patients, who were initially diagnosed with CUP, the cancer origin was ultimately detected; however, ovarian cancer accounts for only $2-4 \%$ of such cases $(9,12)$. In our cases, adequate sampling of tumor tissues or cells using thoracoscopy and immunohistochemical examinations were useful in the identification of the tumor origin.

Although serum tumor markers can be helpful in certain CUP cases, routine evaluation of commonly used epithelial tumor markers, namely CEA, CA19-9, CA15-3 and CA-125, has no proven diagnostic value (4). With regard to ovarian cancer, 

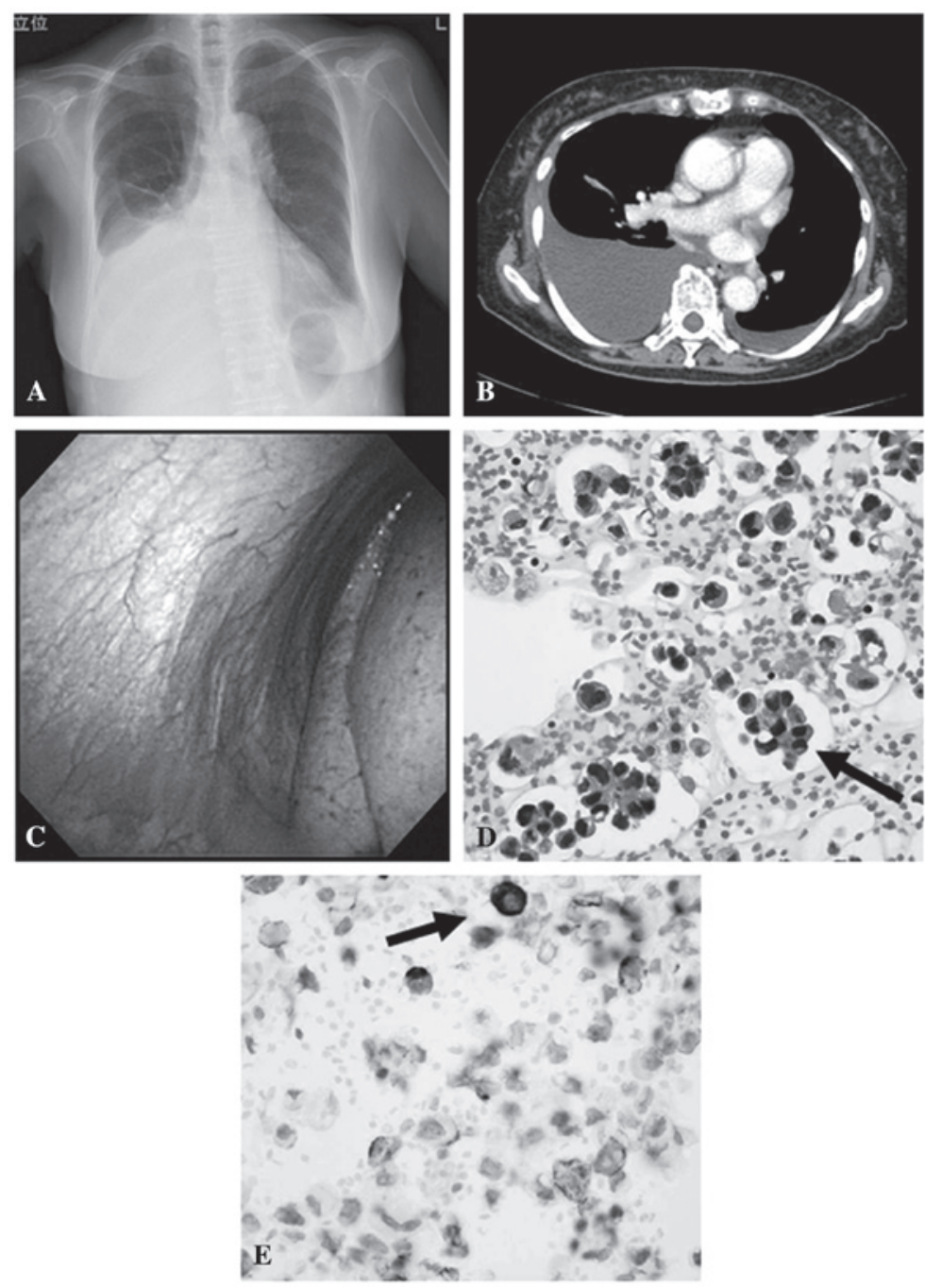

Figure 3. Case 3. (A) Chest X-ray film showing right pleural effusion. (B) CT scan showing bilateral pleural effusion. (C) Thoracoscopic image showed no lesion of the parietal pleura. (D) Pleural fluid cell block indicating adenocarcinoma. Hematoxylin and eosin staining (x400). (E) Immunohistochemical examination of the pleural fluid cell block showed positivity for CA-125 (x400).

almost $80 \%$ of cases exhibit elevated serum CA-125 levels, but the specificity of this measurement is insufficient for the diagnosis $(11,13)$. A recent report described an association between increased KL-6 levels and ovarian cancer, therefore, KL-6 is a useful tumor marker for ovarian cancer (14).

KL-6 is a circulating high-molecular-weight glycoprotein classified as MUC1 mucin, and is widely accepted as a marker for interstitial lung disease (15-17). Originally, KL-6 was introduced as a tumor marker for lung cancer, and elevated serum KL-6 levels were observed in 30-60\% patients with lung adenocarcinoma, pancreatic cancer and breast cancer (15). Furthermore, in ovarian cancer cases, approximately $38 \%$ of patients exhibited increased levels of serum KL-6 (14). Although the mechanism of serum KL- 6 elevation in cancer patients is not clarified, serum KL-6 is suspected to be derived from cancer cells $(14,16)$. In a previous report, the serum levels of KL-6 and CA-125 were correlated with each other and they indicated the state of disease in patients with advanced and/ or relapsed phase ovarian cancer (14). These phenomena were also observed in our cases; therefore, KL- 6 is believed to be a useful tumor marker for diagnosing advanced ovarian cancers.
Further investigations of our findings are warranted, and the biological mechanisms should be evaluated in detail.

In summary, we describe 3 cases of ovarian cancer with plural effusion diagnosed by thoracoscopy, including medical thoracoscopy. Although the early diagnosis of ovarian cancer was difficult in our cases, the measurement of serum KL-6 and CA-125 levels and immunohistochemical analyses should have been useful. Our report may provide additional information on the clinical significance of medical thoracoscopy and measurement of serum KL-6.

\section{References}

1. McLean AN, Bicknell SR, McAlpine LG and Peacock AJ: Investigation of pleural effusion: an evaluation of the new Olympus LTF semiflexible thoracofiberscope and comparison with Abram's needle biopsy. Chest 114: 150-153, 1998.

2. Kaburagi T, Kuroda $\mathrm{H}$ and Amemiya R: Thoracoscopy under local anesthesia focusing on findings of carcinomatous pleurisy. Kikanshigaku (J Jpn Society Bronchology) 26: 326-330, 2004.

3. Canto A, Rivas J, Saumench J, Morera R and Moya J: Points to consider when choosing a biopsy method in cases of pleurisy of unknown origin. Chest 84: 176-179, 1983. 
4. Pavlidis N, Briasoulis E, Hainsworth J and Greco FA: Diagnostic and therapeutic management of cancer of an unknown primary. Eur J Cancer 39: 1990-2005, 2003.

5. Casal RF, Eapen GA, Morice RC and Jimenez CA: Medical thoracoscopy. Curr Opin Pulm Med 15: 313-320, 2009.

6. Narushima M, Matsuishi J, Yamashita J and Suzuki H: Current situations of medical thoracoscopy for pleural deseases in Japan. Kikanshigaku (J Jpn Society Bronchology) 26: 711-716, 2004.

7. Johnston WW: The malignant pleural effusion. A review of cytopathologic diagnoses of 584 specimens from 472 consecutive patients. Cancer 56: 905-909, 1985.

8. Kobayashi Y, Terauchi F, Nishi H, Fujitou A, Itou H and Isaka K: A case of advanced ovarian cancer upstaged on the bases of pleural washing cytology. J Obstet Gynaecol Res 35: 588-592, 2009.

9. Fukuoka T, Matsuoka E, Chiba S, Takayama S and Ohono S: Ovarian cancer that was initially diagnosed as malignant pleural effusion of unknown primary origin. J Rural Med 4: 41-44, 2009.

10. Chernow B and Sahn SA: Carcinomatous involvement of the pleura: an analysis of 96 patients. Am J Med 63: 695-702, 1977.

11. Cannistra SA: Cancer of the ovary. N Engl J Med 351: 2519-2529, 2004.

12. Nystrom JS, Weiner JM, Heffelfinger-Juttner J, Irwin LE, Bateman JR and Wolf RM: Metastatic and histologic presentations in unknown primary cancer. Semin Oncol 4: 53-58, 1977.
13. Topalak O, Saygili U, Soyturk M, et al: Serum, pleural effusion, and ascites CA-125 levels in ovarian cancer and nonovarian benign and malignant diseases: a comparative study. Gynecol Oncol 85: 108-113, 2002.

14. Matsunaga T, Hiraiwa T, Kodaira H and Imai K: KL-6 is useful tumor marker for ovarian cancer. Nihon Fujinka Shyuyou Gakkai Zasshi (Jpn J Gynecol Oncol) 26: 387-393, 2008.

15. Kohno N, Akiyama M, Kyoizumi S, Hakoda M, Kobuke K and Yamakido M: Detection of soluble tumor-associated antigens in sera and effusions using novel monoclonal antibodies, KL-3 and KL-6, against lung adenocarcinoma. Jpn J Clin Oncol 18: 203-216, 1988.

16. Miyazaki K, Kurishima K, Kagohashi K, et al: Serum KL-6 levels in lung cancer patients with or without interstitial lung disease. J Clin Lab Anal 24: 295-299, 2010.

17. Ohnishi H, Yokoyama A, Kondo K, et al: Comparative study of KL-6, surfactant protein-A, surfactant protein-D, and monocyte chemoattractant protein-1 as serum markers for interstitial lung diseases. Am J Respir Crit Care Med 165: 378-381, 2002. 